\title{
Impact of Soft Contact Lens Edge Design and Midperipheral Lens Shape on the Epithelium and Its Indentation With Lens Mobility
}

\author{
James S. Wolffsohn, ${ }^{1}$ Thomas Drew, ${ }^{1,2}$ Sandeep Dhallu, ${ }^{1}$ Amy Sheppard, ${ }^{1}$ Greg J. Hofmann, ${ }^{3}$ \\ and Mark Prince ${ }^{2}$ \\ ${ }^{1}$ Ophthalmic Research Group, Life and Health Sciences, Aston University, Birmingham, United Kingdom \\ ${ }^{2}$ Biomechanical Research Group, Engineering and Applied Sciences, Aston University, Birmingham, United Kingdom \\ ${ }^{3}$ Johnson and Johnson Visioncare, Jacksonville, Florida
}

Correspondence: James S. Wolffsohn, Life and Health Sciences, Aston University, Aston Triangle, Birmingham, B4 7ET, UK;

j.s.w.wolffsohn@aston.ac.uk.

Submitted: May 17, 2013

Accepted: August 3, 2013

Citation: Wolffsohn JS, Drew T, Dhallu S, Sheppard A, Hofmann GJ, Prince M. Impact of soft contact edge lens design and midperipheral lens shape on the epithelium and its indentation with lens mobility. Invest Ophthalmol Vis Sci. 2013;54:6190-6196. DOI: $10.1167 /$ iovs.13-12425
Purpose. To evaluate the influence of soft contact lens midperipheral shape profile and edge design on the apparent epithelial thickness and indentation of the ocular surface with lens movement.

Methods. Four soft contact lens designs comprising of two different plano midperipheral shape profiles and two edge designs (chiseled and knife edge) of silicone-hydrogel material were examined in 26 subjects aged $24.7 \pm 4.6$ years, each worn bilaterally in randomized order. Lens movement was imaged enface on insertion, at 2 and 4 hours with a high-speed, high-resolution camera simultaneous to the cross-section of the edge of the contact lens interaction with the ocular surface captured using optical coherence tomography (OCT) nasally, temporally, and inferiorly. Optical imaging distortions were individually corrected for by imaging the apparent distortion of a glass slide surface by the removed lens.

RESults. Apparent epithelial thickness varied with edge position $(P<0.001)$. When distortion was corrected for, epithelial indentation decreased with time after insertion $(P=0.010)$, changed after a blink $(P<0.001)$, and varied with position on the lens edge $(P<0.001)$, with the latter being affected by midperipheral lens shape profile and edge design. Horizontal and vertical lens movement did not change with time postinsertion. Vertical motion was affected by midperipheral lens shape profile $(P<0.001)$ and edge design $(P<0.001)$. Lens movement was associated with physiologic epithelium thickness for lens midperipheral shape profile and edge designs.

Conclusions. Dynamic OCT coupled with high-resolution video demonstrated that soft contact lens movement and image-corrected ocular surface indentation were influenced by both lens edge design and midperipheral lens shape profiles.

Keywords: contact lenses, epithelial thickness, epithelial indentation, edge design
$\mathrm{C}$ ontact lenses need to move over the ocular surface with each blink to allow tear exchange and a healthy ocular physiology to be maintained. ${ }^{1-3}$ Greater corneal and conjunctival fluorescein staining have been found with poorly fitting lenses and higher levels of bulbar and limbal hyperaemia with loose fitting lenses. ${ }^{4}$ In addition, fitting characteristics can affect lens comfort and quality of vision. ${ }^{5}$ With an average blink rate of approximately 12 times each minute, a soft contact lens oscillates across the ocular surface approximately 11,500 times a day, covering a distance of approximately $7 \mathrm{~m} .{ }^{6}$ Highresolution optical coherence tomography (OCT) can be used to image the interaction between the edge of the contact lens and the corneal epithelium. ${ }^{7}$ It has recently been shown using static imaging with high-resolution OCT that contact lenses with a rounded edge worn for 30 minutes appear to indent the corneal epithelium more and underlying post lens gaps near the corneal scleral junction were more common than that caused by lenses with angular edges. ${ }^{8}$ Even apparently well-fitting soft contact lenses can impact the ocular surface and corneoscleral morphology, ${ }^{9}$ which might be linked with comfort and future complications in some individuals. However, the indentation in this previous study was only subjectively graded and commercially available contact lenses were used differing in lens material, so the effects of lens edge and shape profile were confounded. ${ }^{8}$

Contact lens parameters are traditionally selected based on central corneal curvature measured with a keratometer and the horizontal visible iris diameter assessed using a ruler. ${ }^{10}$ However, soft contact lenses drape over the entire cornea, across the limbus, and onto the bulbar conjunctiva. As a result, peripheral corneal topography and the corneoscleral junction profile determined by OCT have been shown to contribute to the prediction of lens fit. ${ }^{11}$ Hence, the mismatch between contact lens design profile and underlying ocular surface shape should be considered in conjunction to the lens interaction with the corneal epithelium. Any indentation of the epithelium is a dynamic phenomenon, driven by the interaction between the contact lens movement, edge thickness profile, eyelid tautness, and muscular force. Hence, any change in indentation with time, together with the lens movement dynamics, need to 
TABle 1. Contact Lens Design Parameters

\begin{tabular}{ll}
\hline \multicolumn{1}{c}{ Property } & \multicolumn{1}{c}{ Range } \\
\hline Material & Senofilcon A \\
Modulus & $74-118 \mathrm{psi}$ \\
Tensile strength & $38-156 \mathrm{psi}$ \\
Elongation & $80-339 \%$ \\
Toughness & $>24 \mathrm{in} \# / \mathrm{in}^{3}$ \\
Water content & $36-40 \%$ \\
Dk, edge corrected & $103 \pm 21$ \\
& $\left(10^{-11}\left[\mathrm{~cm}^{2} / \mathrm{sec}\right]\left[\mathrm{mlO}_{2} / \mathrm{mL} \times \mathrm{mm} \mathrm{Hg]}\right)\right.$ \\
Refractive index & $1.42 \pm 0.02$ \\
Diameter & $14.00 \pm 0.20 \mathrm{~mm}$ \\
Center thickness & $0.070 \pm 0.017 \mathrm{~mm}$ \\
\hline
\end{tabular}

in\#, inch-pound-force; Dk, oxygen permeability.

be considered. Finally, other OCT imaging studies that have observed the interaction between a contact lens and the ocular surface seem to have largely ignored the distortion effects of the contact lens curvature and refractive index on the underlying imaged tissues. This effect will be influenced by hydration of the contact lens and, hence, may vary between individuals. Optical distortion will naturally result in a rounder (thicker) lens edge appearing to indent the corneal epithelium more than an angular (thinner) edge and would magnify any post lens gaps, resulting in them being observed more often, which could explain previous findings. ${ }^{8}$

Therefore, this study combined a high-resolution digital camera to capture contact lens movement with a custom built dynamic swept source OCT to capture the interaction between the edge of the contact lens and the ocular surface. Four experimental lens designs were constructed from the same material to assess the interaction of midperipheral lens shape profile and edge design in vivo. Hence, the independent contribution of lens shape parameters on the conjunctival epithelium, with lens movement could be elucidated

\section{MeTHODS}

The study was approved by the Aston University ethics committee and by the Medicines and Healthcare products
Regulatory Agency (UK) as the contact lenses were investigational products. The research followed the tenets of the Declaration of Helsinki. Signed consent was obtained from each subject following explanation of the study and the possible consequences. Each subject was enrolled in the study if they were an adapted soft contact lens wearer, had refractive astigmatism of no more than 0.75 diopters (D), had a corrected visual acuity of at least $20 / 30$ in each eye, there was no evidence or history of ocular tissue anomaly, ocular surgery, ocular infection or inflammation, dry eye, allergy, or any ocular surface or systemic disease that might have affected the relationship between the contact lens and the ocular surface. None of the subjects were taking ocular medication and all had not worn contact lenses over the week previous to the first visit and did not wear habitual lenses between study visits.

Four different lens designs made from the same senofilcon A material were examined (Table 1). These comprised of two midperipheral lens shape profiles designed to provide a greater lens induced pressure on the ocular surface ( $8.4-\mathrm{mm}$ base curve and thicker, stiffer midperiphery) compared with a flatter (8.8 $\mathrm{mm}$ ) base curve, thinner midperiphery shape profile; two lens edge designs (either a chiseled or knife edge). The contact lens designs had a combination of midperipheral lens shape profile and edge design such that all four design combinations were worn by all subjects (Fig. 1). All lenses had a $0.00 \pm 0.25 \mathrm{D}$ power so that power related thickness profiles did not confound the study design and subjects could wear their spectacles to obtain appropriately corrected vision, if needed.

A novel simultaneous dual imaging technique was developed to assess dynamic contact lens movement and its effect on the ocular surface during blinking. The system comprised of a high-speed swept source domain OCT system and a highresolution, high-speed camera system (HSC). The OCT device consisted of a Santec IVS-2000 (Aichi, Japan) customized to optimize its use in anterior segment imaging. Hardware customization included the addition of a tunable attenuation unit allowing power input to the ocular system to be controlled within eye safe levels. Additional attenuation between the return light path and the balance detector allowed optimization of the signal-to-noise ratio and selectable sensitivity limited the negative effects of dropping output power to eye safe levels. The OCT delivered 20,000 A-scans per
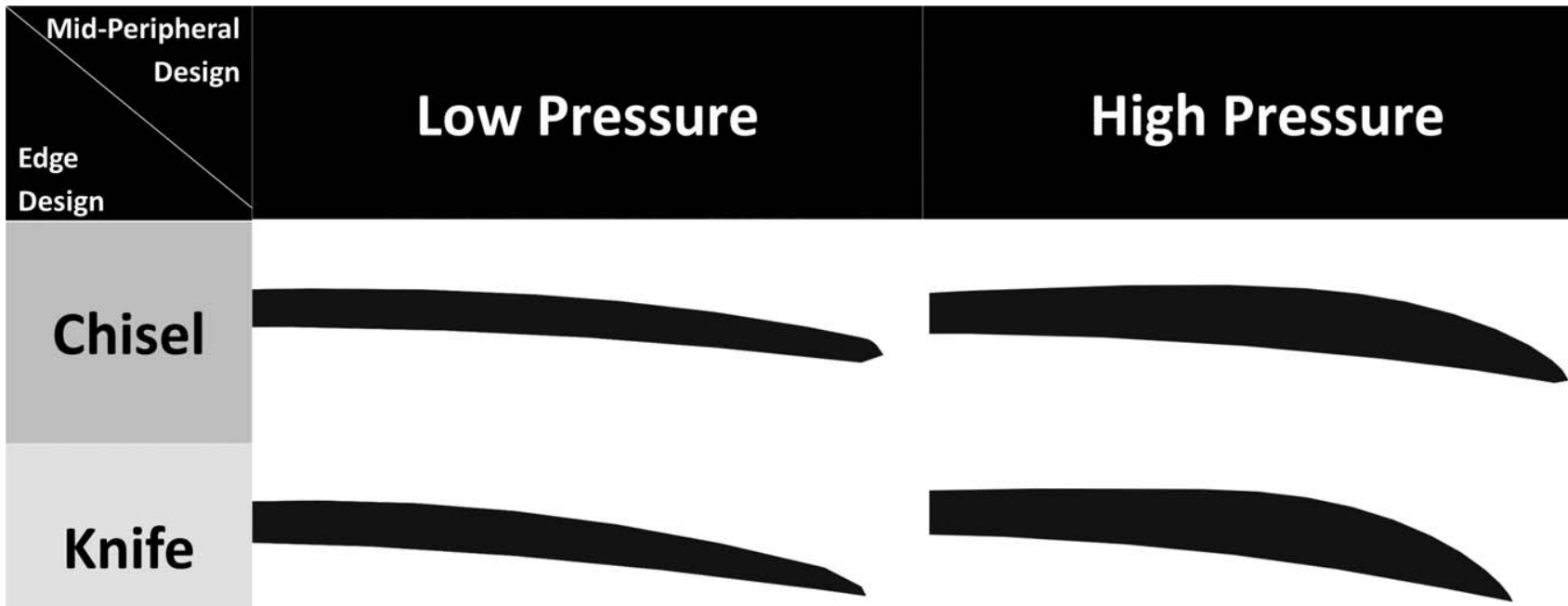

Figure 1. Four different lens designs in senofilcon A material were examined in this study. These comprised of a midperipheral lens profile designed to provide a greater lens-induced pressure on the ocular surface (8.4-mm base curve and thicker, stiffer midperiphery; high pressure) compared with a flatter $(8.8 \mathrm{~mm}$ ) base curve, thinner midperiphery shape profile (low pressure), in combination with a chisel or knife lens edge designs. All four design combinations were worn by all subjects. 
second giving a frame rate of 27 frames per second (B-scans) with an axial resolution of $7 \mu \mathrm{m}$ and a lateral resolution of 23 $\mu \mathrm{m}$. Scan range was selectable between 1 and $15 \mathrm{~mm}$. Scanning could be performed in any meridian and was changeable through software in real time by $1^{\circ}$ intervals. The bespoke alignment system and headrest also allowed both the OCT and HSC to share a common focal point and image simultaneously. The imaging probe was mounted on a slit-lamp base with additional fine alignment from a 3-axis stage and vertical tilt platform. This allowed the imaging head to be positioned perpendicular to the ocular or surface of the contact lens at the point of highest sensitivity while keeping the HSC imaging collinear with respect to the optical axis of the eye.

The HSC comprised of the Flacon 4M60 camera (Teledyne DALSA, Thousand Oaks, CA) accompanied by a CameraLink frame grabber NI PCIe-1429 (National Instruments, Austin, TX) delivering $2352 \times 1728$ pixels with a capture speed up to 62 frames per second. A custom optical system from Schneider Optics (Hauppauge, NY) captured a $16 \mathrm{~mm}^{2}$ field of view at a resolution of $16 \mu \mathrm{m}$. A fixation target consisting of a light emitting diode (LED) kept the subjects concentration fixed throughout scanning and allowed rapid eye alignment. Ocular illumination was provided by a bespoke array of broad spectrum white LEDs. The contact lenses were imprinted with an opaque centered circle during the manufacturing process to allow lens centration and movement to be visualized by the HSC.

Custom software was developed using Labview (National Instruments) to control the OCT system and HSC system as well as to maintain precise timing synchronization between the two platforms. The system relied on parallel processing techniques due to the high data throughput of the system. A novel system was developed allowing the HSC image data to be buffered in real time directly to the computers random access memory (RAM) bypassing the normal method where data are written directly to the hard disk drive, allowing high image capture rates.

The study was a randomized, bilateral, cross-over, nondispensing study, in which subjects wore each contact lens type for four hours. Each lens type was worn in both eyes on a different visit, separated by at least one day, with subjects refraining from contact lens wear between visits. The measurements analyzed were taken from the right eye only. The study was conducted in a consulting room with controlled temperature $\left(20 \pm 2^{\circ} \mathrm{C}\right)$ and humidity $(40 \pm 5 \%)$. All subjects were scheduled after $10 \mathrm{AM}$ to avoid corneal edema and the alteration of the tear film induced by sleep, which could have affected the results. ${ }^{12,13}$ Twenty-six subjects (average age 24.7 $\pm 4.6,14$ female) completed the study. Eye health was examined with a slit-lamp biomicroscope. The randomly selected contact lens type was fitted to both eyes and the lens movement was imaged enface immediately on insertion at 2 and 4 hours postinsertion with the HSC; simultaneously, the cross-section of the edge of the contact lens interaction with the ocular surface was captured by the OCT (scanning perpendicularly). At least three blinks were captured with the HSC and OCT systems and the two most similar were analyzed. This was repeated at the 3 (nasal), 6 (inferior), and 9 (temporal) o'clock lens edge positions in random order.

\section{Data Analysis}

System calibration and dewarping was performed with a series of known precision reference spheres (Grade 10 stainless steel ball bearings with a diameter tolerance of $1.3 \mu \mathrm{m}$ ) with diameters of 7, 16, and $24 \mathrm{~mm}$ (Simply Bearings Ltd., Lancashire, UK), and conversion factors were applied in software (axial resolution 141 pixels per millimeter). The errors of measure- ment were $\pm 0.1 \mu \mathrm{m}$ in the axial (vertical) plane and $\pm 1.4 \mu \mathrm{m}$ in the lateral plane. Each individual's contact lenses were imaged using the OCT system on a glass slide immediately upon removal (within 10 seconds), after the last on-eye scan to allow the distortion of the underlying structures caused by the contact lens to be measured and compensated for (Fig. 2). As the lens was essentially flat over the small area of the peripheral contact lens imaged by the OCT, differences in lens and ocular surface curvature between the in vivo and extracted lens on the glass slide were negligible. It was confirmed in a pilot study that the distortion of the surface of a glass slide by imaging through a contact lenses worn for 5 minutes or 4 hours was the same. Hence, the distortion calculated for each individual after 4 hours wear was used to compensate their image data at each time point with that contact lens.

Further OCT image processing was performed with Matlab (The Mathworks Inc., Natick, MA) by resampling the raw OCT intensity output and applying a custom rescaling algorithm to rescale the peak reflections over the 16-bit image space, giving a superior dynamic range (contrast) and reducing high frequency speckle (noise) compared with a standard OCT system output. A semi-automated image processing suite was developed using Labview (National Instruments) allowing apparent epithelium thicknesses, lens thickness, and indentation depths to be measured from the OCT data (Fig. 2). The lens edge was identified subjectively and once the program had corrected the image for curvature, the objectively located interface points were confirmed by the masked researcher.

The HSC data was analyzed with distance between the centre of a circle imprinted on the contact lens during the manufacturing process and center of the limbus calculated in each frame to assess lens horizontal and vertical movement postblink and the damping coefficient $(\lambda)$ defined by the exponential equation:

$$
N(t)=N_{0} \mathrm{e}^{-\lambda t}
$$

where $N=$ position, $0=$ immediately after a blink, and $t=$ time.

As the lens movement and indentation data were normally distributed, a repeated measure ANOVA was conducted with time after insertion, position of imaging the lens edge, lens profile, and pre- and postblink as factors, with the data averaged across two blinks. This approach limits the number of analyses conducted, minimizing the risk of type I statistical error. The sample size met the requirements for sufficient replicates for a repeated measures design. ${ }^{14}$

\section{Results}

\section{Apparent Epithelial Thickness}

Apparent epithelial thickness was significantly thinner $1 \mathrm{~mm}$ from the edge under the lens $(55.8 \pm 14.3 \mu \mathrm{m})$ than $1-\mathrm{mm}$ beyond the leading lens edge $(66.4 \pm 17.2 \mu \mathrm{m} ; F=88.815, P<$ 0.001 ), but the interaction between the apparent epithelial thickness at these two locations and time after lens insertion, lens position, during the blink and lens design were consistent, so data for the average apparent epithelial thickness under and beyond the lens edge is presented. Apparent epithelial thickness remained constant over time, but varied with position on the lens edge (Table 2). There was no significant difference immediately after a blink to immediately before the next blink, although there was an interaction with lens edge profile (knife edge before to after a blink $61.4 \pm 17.6$ vs. 61.3 $\pm 16.8 \mu \mathrm{m}$; chisel edge $60.2 \pm 15.9$ vs. $61.5 \pm 16.5 \mu \mathrm{m} ; F=$ $7.408, P=0.008$ ). There was an interaction between position on the lens edge and change in apparent epithelial thickness immediately after a blink to before the next blink $(F=3.921, P$ 


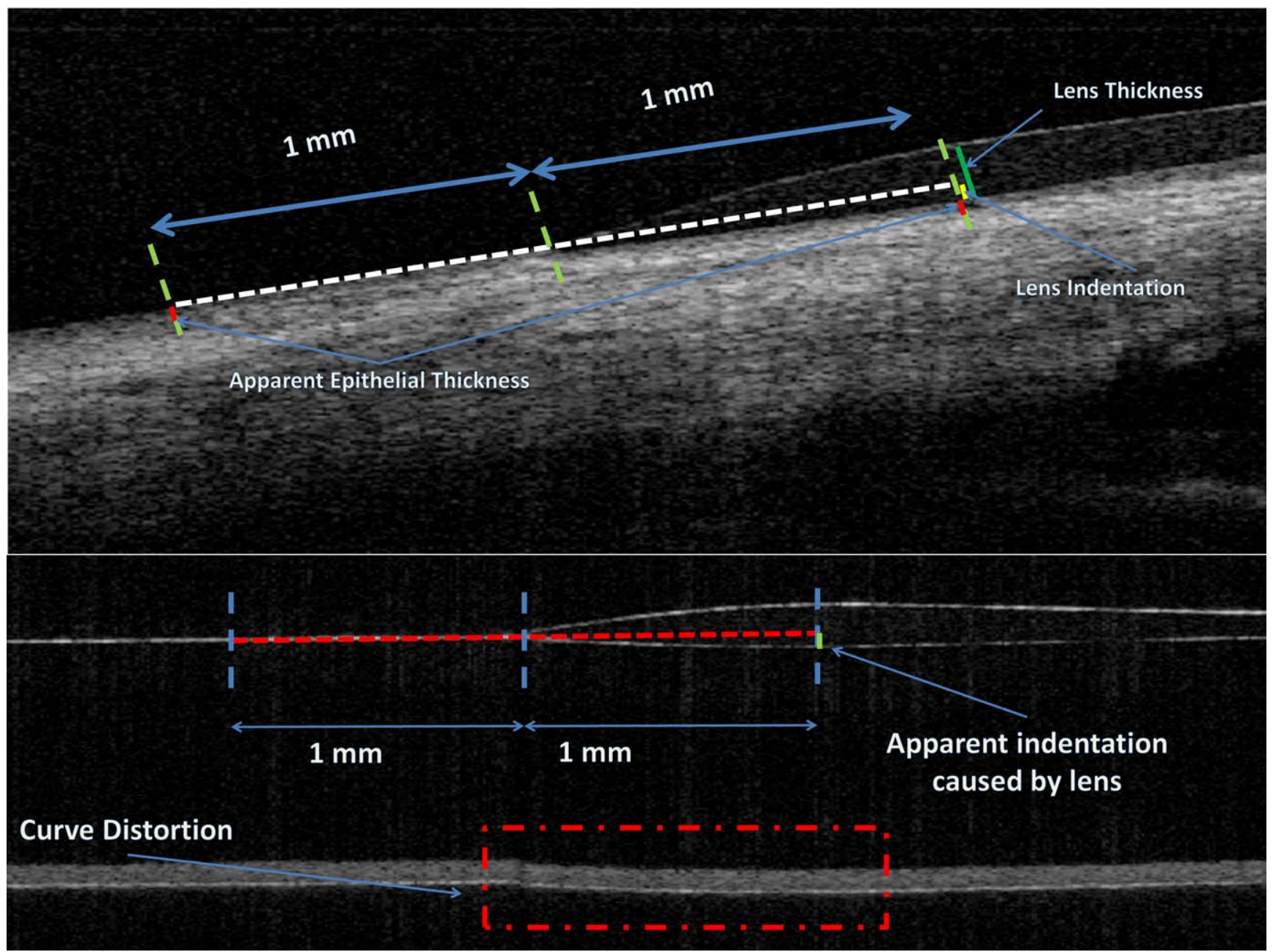

Figure 2. Image analysis of dewarped, calibrated, and resampled OCT images to determine apparent epithelial indentation. The upper image shows the lens on the ocular surface: the apparent epithelial thickness was assess 1-mm beyond and under the lens edge; indentation was assessed 1-mm under the lens edge as the distance between a straight line fitted to the 1-mm beyond the leading lens edge and the perpendicular position of the epithelium 1-mm under the lens; the lens thickness was measured 1-mm from the lens edge. The lower image shows the same lens immediately after removal from the eye placed on a glass slide: the optical curvature and refractive index of the lens distorts the appearance of the flat slide, allowing the distortion 1-mm under the lens edge (caused by the same hydrated lens and the individual's tear film refractive index) to be quantified and subtracted from the apparent indentation of the lens on the ocular surface to determine true indentation.

$=0.022$ ). There was also an interaction between the lens edge profile, the difference in apparent epithelial thickness between under and beyond the lens edge and time after contact lens insertion $(F=3.204, P=0.044)$, and with the additional variable of position of measurement along the lens edge $(F=$ $3.760, P=0.005)$. None of the other interactions were statistically significant $(P>0.05)$.

\section{Lens Thickness}

Lens thickness $1 \mathrm{~mm}$ from the lens edge differed between the midperipheral lens shape profiles (low pressure: 104.1 \pm 11.1 $\mu \mathrm{m}$ versus high pressure design: $146.5 \pm 13.9 \mu \mathrm{m} ; F=$ 803.079, $P<0.001)$, but not with edge design $(F=0.053, P=$ $0.818)$, time after contact lens insertion $(F=2.630, P=0.076)$, lens edge position $(F=0.890, P=0.413)$, or pre- and postblink $(F<0.001, P=0.993)$, and there was no interaction between these variables $(P>0.05)$.

\section{Indentation}

When indentation was corrected for distortion, as evidenced from the glass slide, epithelial indentation decreased with time, varied with position on the lens edge, and changed after a blink (Table 2). Indentation did not vary with midperipheral lens shape profile or with edge design, as it appeared to do if optical distortion was not compensated for (low pressure $52.3 \pm 18.8$ $\mu \mathrm{m}$, high pressure design $61.2 \pm 22.9 \mu \mathrm{m}, F=17.567, P<$ 0.001 ; knife edge $59.1 \pm 22.4 \mu \mathrm{m}$, chisel edge $54.7 \pm 20.2 \mu \mathrm{m}$, $F=4.916, P=0.030$ ). There were significant interactions between position on the lens edge with midperipheral lens shape profile $(F=3.066, P=0.049)$ and with the edge design $(F$ $=3.364, P=0.037)$, change after a blink with midperipheral lens shape profile $(F=31.276, P<0.001)$, and position on the lens edge with change after a blink $(F=5.500, P=0.005)$. None of the other interactions were statistically significant $(P>0.05)$.

\section{Lens Movement}

Horizontal lens movement did not change with time postinsertion $(F=0.690, P=0.503)$ and was not affected by midperipheral lens shape profile (low pressure: $0.55 \pm 0.16$ $\mathrm{mm}$; high pressure: $0.53 \pm 0.16 \mathrm{~mm} ; F=0.660, P=0.419)$, or by edge design (knife: $0.53 \pm 0.17 \mathrm{~mm}$; chisel: $0.55 \pm 0.16 \mathrm{~mm}$; $F=1.660, P=0.090$ ), and there were no significant interactions 
TABLE 2. Average Apparent Epithelial Thickness and Indentation (Corrected for Optical Distortion) of the Epithelial Surface Beyond and Under the Lens Edge (Averaged) With Time After Lens Insertion, Position on the Lens Edge, Blink, and Contact Lens

\begin{tabular}{|c|c|c|}
\hline & Apparent Epithelial Thickness, $\mu \mathrm{m}$ & Indentation, $\mu \mathrm{m}$ \\
\hline \multicolumn{3}{|l|}{ Time after lens insertion } \\
\hline On insertion & $60.5 \pm 17.0$ & $8.9 \pm 2.5$ \\
\hline 2 hours wear & $61.9 \pm 17.7$ & $5.3 \pm 2.4$ \\
\hline 4 hours wear & $60.9 \pm 15.7$ & $3.8 \pm 2.5$ \\
\hline Significance & $F=2.193, P=0.115$ & $F=4.731, P=0.010$ \\
\hline \multicolumn{3}{|l|}{ Lens position } \\
\hline 3 o'clock, nasal & $63.6 \pm 17.2$ & $2.8 \pm 23.6$ \\
\hline 6 o'clock, inferior & $64.6 \pm 16.6$ & $7.1 \pm 23.9$ \\
\hline 9 o'clock, temporal & $55.0 \pm 14.4$ & $14.0 \pm 23.2$ \\
\hline Significance & $F=44.642, P<0.001$ & $F=59.180, P<0.001$ \\
\hline \multicolumn{3}{|l|}{ Blink } \\
\hline Immediately after & $60.7 \pm 16.8$ & $7.8 \pm 24.8$ \\
\hline Before next & $61.4 \pm 16.7$ & $4.3 \pm 24.2$ \\
\hline Significance & $F=3.675, P=0.059$ & $F=31.276, P<0.001$ \\
\hline \multicolumn{3}{|c|}{ Midperipheral lens design } \\
\hline Low pressure & $60.9 \pm 15.6$ & $6.1 \pm 21.3$ \\
\hline High pressure & $61.3 \pm 17.8$ & $5.9 \pm 27.4$ \\
\hline Significance & $F=1.227, P=0.272$ & $F=0.026, P=0.872$ \\
\hline \multicolumn{3}{|l|}{ Lens edge design } \\
\hline Knife edge & $61.4 \pm 17.2$ & $7.5 \pm 25.1$ \\
\hline Chisel edge & $60.8 \pm 16.2$ & $4.6 \pm 23.9$ \\
\hline Significance & $F=0.229, P=0.572$ & $F=0.097, P=0.757$ \\
\hline
\end{tabular}

Design; $n=26$.

between parameters $(P>0.05)$. Vertical lens movement was also unaffected by time postinsertion $(F=1.180, P=0.310)$, but was significantly affected by midperipheral lens shape profile (low pressure: $0.79 \pm 0.39 \mathrm{~mm}$; high pressure: $0.68 \pm 0.27$ $\mathrm{mm} ; F=8.130, P<0.001)$ and edge design (knife: $0.64 \pm 0.26$ mm, chisel: $0.82 \pm 0.38 \mathrm{~mm} ; F=22.500, P<0.001)$, but there were no significant interactions between these parameters $(P>$ 0.05). Damping of the lens movement was not affected by time postinsertion $(F=0.720, P=0.488)$ or midperipheral lens shape profile (low pressure $0.18 \pm 0.23$; high pressure design $0.15 \pm$ $0.18 ; F=1.120, P=0.291)$. However, edge design was a significant factor (knife: $0.09 \pm 0.16$; chisel: $0.24 \pm 0.22 ; F=$ 41.730, $P<0.001)$. There were no significant interactions between the parameters $(P>0.05)$.

\section{Relationship Between Lens Movement and OCT Parameters}

The relationship between apparent epithelial thickness under the lens edge and beyond the leading edge of the contact lens, and the corrected indentation caused by the lens for the midperipheral lens shape profiles and chisel and knife edge designs was investigated (Table 3). Greater horizontal lens movement was associated with a thicker physiologic epithelium beyond the leading lens edge with the knife edge design and for the low pressure midperipheral profile lenses. Greater vertical movement was correlated with thicker physiologic epithelium beyond the leading lens edge for all but the low pressure midperipheral profile lenses (Table 3).

\section{Discussion}

This study, for the first time, coupled high-resolution OCT with high-speed, enface video to examine in detail the lens-eye interaction, focused on the lens edge. The interaction between the ocular surface and the contact lens, especially around the lens edge, is of key interest in maintaining the health of the eye in contact lens wear and in terms of optimizing lens comfort. ${ }^{1,4}$ Characterization of the soft contact lens interaction with the ocular surface should enhance our understanding of current lens design parameters and allow improved designs to be generated. The repeated measure design with each subject wearing the two lens edge designs and two midperipheral designs of lenses, all made of the same contact lens material, overcomes any confounding influence from any differences between subjects such as in eyelid dynamics, ocular surface shape profile, and corneal thickness.

Lens thickness $1 \mathrm{~mm}$ from the lens edge confirmed the difference between the midperipheral shape lens profile designs and was not affected by edge design. There were no other interactions with lens thickness, confirming the consistent repeatability of OCT imaging, and suggesting that any hydration changes and/or lens squeezing changes with time are minimal in this portion of the contact lens. ${ }^{15}$

Apparent epithelial thickness with the bespoke dynamic OCT system designed for this study was similar to that reported previously using a fourier domain OCT and also that found in the periphery of the cornea measured with ultrasound. ${ }^{16,17}$ Apparent epithelial thickness remained constant over time, but varied with position on the lens edge, with the 9 o'clock (temporal) position being thinner than at the 3 or 6 o'clock (nasal and inferior) positions. The thicker apparent epithelial thickness in the nasal and inferior quadrants has been shown previously by Hall and colleagues and relates to the differences in corneal topography in the corneoscleral region. ${ }^{11}$ This difference was not evident, though in a small group of subjects after lens wear. ${ }^{9}$ Both lens midperipheral and edge design in another previous study had no impact on indentation or apparent epithelial thickness, unlike the findings of Shen and colleagues. $^{8}$ This may have in part been due to the lens indentation correction applied in this study, as without image 
TaBle 3. The Correlation Between the Apparent Epithelial Thickness Under the Lens Edge ("Under Lens") and Below the Leading Edge ("BeyondLens") of the Contact Lens, and the Corrected Indentation Caused by the Lens for the Midperipheral Lens Shape Profile (Low and High Pressure) and Lens Edge Designs (Chisel and Knife Edge) Investigated

\begin{tabular}{|c|c|c|c|c|c|}
\hline \multirow{2}{*}{\multicolumn{2}{|c|}{ Contact Lens Design }} & \multirow{2}{*}{$\begin{array}{c}\text { Contact Lens } \\
\text { Movement }\end{array}$} & \multicolumn{2}{|c|}{ Apparent Epithelial Thickness } & \multirow[b]{2}{*}{ Epithelial Indentation } \\
\hline & & & Beyond Lens & Under Lens & \\
\hline \multirow[t]{6}{*}{ Edge } & Knife & Horizontal & $r=0.473 ; P<0.001^{*}$ & $r=0.254 ; P=0.072$ & $r=-0.347 ; P=0.013^{*}$ \\
\hline & & Vertical & $r=0.313 ; P=0.026^{*}$ & $r=-0.041 ; P=0.775$ & $r=-0.232 ; P=0.101$ \\
\hline & & Damping & $r=0.093 ; P=0.522$ & $r=0.006 ; P=0.968$ & $r=-0.131 ; P=0.359$ \\
\hline & Chisel & Horizontal & $r=0.110 ; P=0.437$ & $r=-0.034 ; P=0.809$ & $r=0.058 ; P=0.684$ \\
\hline & & Vertical & $P=0.370 ; P=0.007^{*}$ & $r=0.220 ; P=0.117$ & $r=-0.061 ; P=0.669$ \\
\hline & & Damping & $r=0.119 ; P=0.401$ & $r=0.145 ; P=0.305$ & $r=0.154 ; P=0.274$ \\
\hline \multirow{6}{*}{$\begin{array}{l}\text { Midperipheral lens } \\
\text { shape profile }\end{array}$} & High pressure: & Horizontal & $r=0.258 ; P=0.065$ & $r=0.111 ; P=0.433$ & $r=-110 ; P=0.437$ \\
\hline & steeper/thicker & Vertical & $r=0.356 ; P=0.010^{*}$ & $r=0.274 ; P=0.050^{*}$ & $r=-0.190 ; P=0.178$ \\
\hline & & Damping & $r=0.043 ; P=0.762$ & $\mathrm{r}=0.165 ; P=0.241$ & $r=-0.038 ; P=0.791$ \\
\hline & Low pressure: & Horizontal & $r=0.344 ; P=0.014^{*}$ & $r=0.107 ; P=0.455$ & $r=-0.051 ; P=0.722$ \\
\hline & flatter/thinner & Vertical & $r=0.262 ; P=0.063$ & $r=0.047 ; P=0.742$ & $r=-0.288 ; P=0.040^{*}$ \\
\hline & & Damping & $r=0.085 ; P=0.555$ & $r=0.027 ; P=0.849$ & $r=0.003 ; P=0.983$ \\
\hline
\end{tabular}

$n=26$.

* Bold denotes a significant result with $P<0.05$.

correction, lens indentation appeared to vary with midperipheral lens shape profile. The finding without image correction suggests a thinner epithelium occurs with a rounder (chisel) edge, converse to the less conjunctival build-up (localized thickness change due to edge pressure) with round compared with angled edges reported in the previous study, but in that study indentation was graded in $25 \%$ steps and the lenses differed in diameter, base curve, and material as well as edge design.

When the epithelial indentation images were corrected based on the lens design worn and the effects of the individual's lens hydration and tear refractive index, the depth of the indentation decreased by an order of magnitude compared with the uncorrected apparent indentation. Indentation from the contact lens (as none was evident over the duration in between blinking without a contact lens in situ) reduced with time post lens insertion, more over the first 2 hours $(\sim 3.4 \mu \mathrm{m})$ than over the subsequent 2 hours $(\sim 1.5 \mu \mathrm{m})$. This equates to approximately one twentieth of the epithelial tissue depth and is less than the resolution of the OCT, so is only evident through rapid OCT imaging speeds and averaging. Indentation was minimal at the 3 o'clock (nasal) lens edge and twice as great at the 9 o'clock (temporal) lens edge as at the 6 o'clock inferior position. The nasal corneoscleral junction has been shown to have a more acute angle than the other meridians, which might explain why the indentation differs in this meridian. ${ }^{11}$ The epithelium was thinnest temporally so the greater indentation in this meridian equates to approximately $25 \%$ of the epithelial thickness. The reason why this meridian is particularly susceptible to lens indentation is unclear, although the eyelid tends to close in a sweeping motion temporally to nasally and so the significant pressure induced by the eyelid margin ${ }^{18}$ may be greater on the cornea at this location. Although lens design (midperipheral shape profile and edge profile) did not impact on epithelial indentation overall, there was an interaction with the variation between the meridians and the indentation changes between blinks varied with midperipheral lens shape profile. It has been suggested that pressure and friction at the edge of the lens, in combination with the edge design, contribute to staining that is often seen after soft lens removal, ${ }^{4,19}$ conjunctival epithelial flaps, ${ }^{20}$ and conjunctival folds ${ }^{21}$ in eyes with a healthy ocular surface. However, the lack of difference in indentation between lens designs found in this parameter controlled study lends minimal support for these hypotheses, at least with this lens material and the design parameters investigated.

The reduction in lens indentation with time did not influence lens movement as the latter remained consistent over the three time periods, although lens movement is known to change over the initial hour of lens wear. ${ }^{6,22,23}$ As well as the expected vertical movement, which was of an order of magnitude expected for similar hydroxy-ethyl methacrylate (HEMA) soft lenses, ${ }^{8}$ there was considerable horizontal slippage (equating to $78 \pm 21 \%$ of the vertical magnitude). Horizontal lens movement was unaffected by the lens design parameters investigated, whereas vertical lens movement was less with the steep, thicker profile designed to induce high pressure and the knife edge design, the latter also resulting in a slower rate of change (damping). The steeper base curve and thicker midperipheral shape profile lens was hypothesized to provide a greater lens-induced pressure (pressure exerted on the eye by the lens) through the decreased base curve and stiffer periphery, resulting in less movement due to higher, normal forces and, hence, greater friction. ${ }^{24}$ This was found to be the case, but the difference related to only approximately $15 \%$ of the total lens movement supporting the clinical observation of modern soft contact lens designs that base curve alone has little influence on lens movement, ${ }^{25}$ although the lens thickness profile can be a determinate. ${ }^{26}$ The knife edge design was on average approximately $11 \%$ less mobile than the chisel edge design, perhaps due to interaction between the contact lens edge and the lens epithelium, but not enough to induce greater indentation; hence, it is likely to be of little short term clinical significance. However, a thicker physiologic apparent epithelial thickness below the leading lens edge was associated with greater horizontal and vertical lens movement in most cases. It is not possible with the resolution of the OCT system to reliably differentiate tear film under the contact lens from epithelial tissue so it is possible that the apparent increase in sublens apparent epithelial thickness represented greater tear flow, resulting in a more mobile lens.

This study was able to overcome many of the limitations of previous research in allowing changes in the ocular surface to be quantified during contact lens wear, controlling for lens material properties, and design differences between commercially available lenses. However, only one material was studied so the magnitude of the changes could vary with other materials of 
differing modulus. Our OCT system did not have the resolution to differentiate the tear film layer. Hence, tear exchange under the lens, which is likely to be impacted by lens design and its conformity with the ocular surface, could not be investigated. While this would not affect the measurement of lens thickness or indentation, the measurement of epithelial thickness includes the tear layer. Consensus on the tear film suggests the thickness is approximately $3 \mu \mathrm{m}$ on the ocular surface and is estimated to be less than $1-\mu \mathrm{m}$ under the lens. ${ }^{27}$ The only factor to significantly change the epithelial (combined with tear film) thickness (termed apparent epithelial thickness) was lens position (Table 2), where the difference was around $10 \mu \mathrm{m}$, which is more than would be expected from purely a tear film effect.

In conclusion, it is evident that studies that do not correct fully for lens thickness, curvature and hydration effects will grossly overestimate the effects of contact lenses on the ocular surface. However, dynamic changes do occur and these are affected by lens midperipheral shape profile and edge design. This is the first study to objectively quantify these effects. The chisel edge caused greater lens mobility, but without measurable differences in epithelial indentation or thickness, perhaps due to the extra edge bulk and less friction with the ocular surface. A flatter, thinner lens midperipheral shape profile also increased lens mobility. Greater apparent epithelial thickness below the leading lens edge was generally associated with increased movement, which may contribute to the differences in this critical clinical parameter between patients. ${ }^{11}$ Hence, the information gained from this investigation will allow improved modeling of the soft contact lens-eye system, including testing of current movement theories based on tear film expulsion and negative pressure effects, ${ }^{6,15}$ and will inform future lens design. The advances in instrumentation constructed to image the dynamic movement of a contact lens simultaneously with high-resolution, cross-sectional images of the lens-ocular surface interaction will allow these new lens designs to be better understood and may contribute to predicting those patients who will experience physiologic complications with certain lens designs.

\section{Acknowledgments}

The authors thank Lee Hall for his useful comments on the drafting of this paper.

Supported by a grant from Johnson and Johnson Vistakon.

Disclosure: J.S. Wolffsohn, Alcon (F, C), Bausch and Lomb (F), Coopervision (F), Johnson and Johnson (F, C); T. Drew, None; S. Dhallu, None; A. Sheppard, None; G.J. Hofmann, Johnson and Johnson (E); M. Prince, None

\section{References}

1. Young G. Evaluation of soft contact lens fitting characteristics. Optom Vis Sci. 1996;73:247-254.

2. Orsborn GN, Zantos SG. The relationship between lens movement and tear exchange under hydrogel contact lenses. Am J Optom Physiol Opt. 1986;62(suppl):S56.

3. McNamara NA, Polse KA, Brand RJ, Graham AD, Chan JS, McKenney CD. Tear mixing under a soft contact lens: effects of lens diameter. Am J Ophthalmol. 1999;127:659-665.

4. Young G, Coleman S. Poorly fitting soft lenses affect ocular integrity. CLAO J. 2001;27:68-74.

5. Dumbleton KA, Chalmers RL, McNally J, Bayer S, Fonn D. Effect of lens base curve on subjective comfort and assessment of fit with silicone hydrogel continuous wear contact lenses. Optom Vis Sci. 2002;79:633-637.
6. Golding TR, Bruce AS, Gaterell LL, Little SA, Macnamara J. Soft lens movement: effect of blink rate on lens settling. Acta Ophthalmol Scand. 1995;73:506-511.

7. Shen M, Wang MR, Wang J, Yuan Y, Chen RF. Entire contact lens imaged in vivo and in vitro with spectral domain optical coherence tomography. Eye Contact Lens. 2010;2:73-76.

8. Shen M, Cui L, Riley C, Wang MR, Wang J. Characterization of soft contact lens edge fitting using ultra-high resolution and ultra-long scan depth optical coherence tomography. Invest Ophthalmol Vis Sci. 2011;52:4091-4097.

9. Alonso-Caneiro D, Shaw AJ, Collins MJ. Using optical coherence tomography to assess corneoscleral morphology after soft contact lens wear. Optom Vis Sci. 2012;89:16191626.

10. Young G, Schnider C, Hunt C, Efron S. Corneal topography and soft contact lens fit. Optom Vis Sci. 2010;87:358-366.

11. Hall L, Young G, Wolffsohn J, Riley C. The influence of corneoscleral topography on soft contact lens fit. Invest Ophthalmol Vis Sci. 2011;52:6801-6806.

12. Patel S, Bevan R, Farrell JC. Diurnal variation in precorneal tear film stability. Am J Optom Physiol Opt. 1988;65:151-154.

13. Shen M, Wang J, Qu J, et al. Diurnal variation of ocular hysteresis, corneal thickness, and intraocular pressure. Optom Vis Sci. 2008;85:1185-1192.

14. Armstrong RA, Eperjesi F, Gilmartin B. The application of analysis of variance (ANOVA) to different experimental designs in optometry. Ophthalmic Physiol Opt. 2002;22:248-256.

15. Martin DK, Holden BA. Forces developed beneath hydrogel contact lenses due to squeeze pressure. Phys Med Biol. 1986; 30;635-649.

16. Reinstein DZ, Archer TJ, Gobbe M, Silverman RH, Coleman J. Epithelial thickness in the normal cornea: three-dimensional display with Artemis very high-frequency digital ultrasound. $J$ Refract Surg. 2008;24:571-581.

17. Prakash G, Agarwal A, Mazhari AI, et al. Reliability and reproducibility of assessment of corneal epithelium thickness by fourier domain optical coherence tomography. Invest Ophthalmol Vis Sci. 2012;53:2580-2585.

18. Shaw AJ, Collins MJ, Davis BA, Carney LG. Eyelid pressure and contact with the ocular surface. Invest Ophthalmol Vis Sci. 2010;51:1911-1917.

19. Maissa C, Guillon M, Garofalo RJ. Contact lens-induced circumlimbal staining in silicone hydrogel contact lenses worn on a daily wear basis. Eye Contact Lens. 2012;38:16-26.

20. Bergmanson JP, Tukler J, Leach NE, Alabdelmoneam M, Miller WL. Morphology of contact lens-induced conjunctival epithelial flaps: a pilot study. Contact Lens Ant Eye. 2012;35:185188.

21. Tapaszto B, Veres A, Kosina-Hagyo K, Somfai GM, Nemeth J. OCT imaging of lid-parallel conjunctival folds in soft contact lens wearers. Optom Vis Sci. 2011;88:1206-1213.

22. Brennan NA, Lindsay RG, McCraw K, Young L, Bruce AS, Golding TR. Soft lens movement: temporal characteristics. Optom Vis Sci. 1994;71:359-363.

23. Golding TR, Harris MG, Smith RC, Brennan NA. Soft lens movement: effects of humidity and hypertonic saline on lens settling. Acta Ophthalmol Scand. 1995;73:139-144.

24. Jenkins JT, Shimbo M. The distribution of pressure behind a soft contact lens. J Biomech Eng. 1984;106:62-65.

25. Wolffsohn JS, Hunt OA, Basra AK. Simplified recording of soft contact lens fit. Contact Lens Ant Eye. 2009;32:37-42.

26. Conway HD, Richman M. Effects of contact lens deformation on tear film pressures induced during blinking. Am J Optom Physiol Opt. 1982;59:13-20.

27. King-Smith PW, Fink BA, Hill RM, Koelling KW, Tiffany JM. The thickness of the tear film. Curr Eye Res. 2004;29:357-368. 